\title{
Research of piezoelectric acoustic liner
}

\author{
Jibin Liu ${ }^{1}$, Haifeng $\mathrm{Lv}^{2}$, Yannan $\mathrm{Han}^{3}$, Jing $\mathrm{Zhao}^{4}$
}

School of Mechanical Engineering, North University of China, Taiyuan, China

${ }^{2}$ Corresponding author

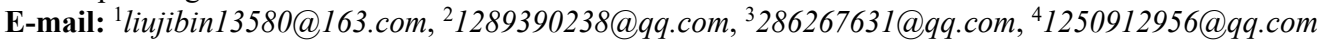

Received 25 September 2017; accepted 2 October 2017

DOI https://doi.org/10.21595/vp.2017.19221

Check for updates

Abstract. The piezoelectric acoustic liner is a new type of acoustic liner that uses piezoelectric patches to replace the traditional mechanical structure. Its working principle is to change the resonator volume of acoustic liner by inverse piezoelectric effect. In this paper, the finite element method is used to analyze the deformation of piezoelectric patches and the acoustic performance of piezoelectric acoustic liner, when the piezoelectric patch deformation is $0.1 \mathrm{~mm}$, the noise elimination frequency band offset of the acoustic liner is about $30 \mathrm{~Hz}$, and related experiments are designed. The experimental results confirm that noise elimination frequency range of piezoelectric acoustic liner is $1100 \mathrm{~Hz}$ to $1300 \mathrm{~Hz}$ within the voltage range of $0 \mathrm{~V}$ to $200 \mathrm{~V}$.

Keywords: acoustic liner, piezoelectric patch, experiment, noise elimination frequency band.

\section{Introduction}

As an effective way to control noise, the application and research of acoustic liner are more and more extensive. The traditional acoustic liner is a series of evenly arranged Helmholtz resonators, but its noise elimination frequency range is narrow [1]. The piezoelectric acoustic liner can be used to change the cavity volume to broaden the noise elimination frequency.

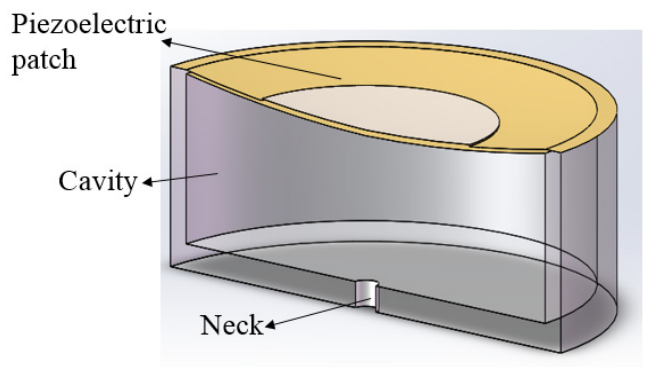

Fig. 1. Working principle diagram of piezoelectric acoustic liner

The main structure of the piezoelectric acoustic liner (Fig. 1) is the Helmholtz resonator, and one of the criteria for evaluating the acoustic performance of Helmholtz resonators is its resonant frequency [2]. The resonant frequency of the resonator can be expressed as:

$f_{0}=\frac{c_{0}}{2 \pi} \sqrt{\frac{\pi r^{2}}{(t+\Delta t) v}}$,

where $t$ and $r$ are the thickness and the radius of the neck, respectively, $\Delta t$ is the sum of the end correction at the inner and outer neck ends, $c_{0}=343 \mathrm{~m} / \mathrm{s}$ is sound velocity in medium, and $v$ is the cavity volume. It can be seen that cavity volume has important influence on the resonant frequency of acoustic liner.

The piezoelectric properties of piezoelectric patches directly affect the volume change of the acoustic liner, which plays an important role in the noise elimination frequency offset of the piezoelectric acoustic liner. Mainly using the inverse piezoelectric effect that the piezoelectric patch will deform when voltage is applied [3]. 


\section{Modeling and simulation}

\subsection{Piezoelectric patch}

Piezoelectric patch consists of substrate and ceramic (Fig. 2), under the same voltage, the deformation of the piezoelectric patch is related to the size of the piezoelectric sheet. Therefore, the finite element method is used to simulate the shape variables of different sizes under different voltages. The DC voltage is loaded on both ends of the piezoelectric ceramic and the displacement constraint is loaded on the edge of the substrate. The structure parameters are shown in the Table 1.

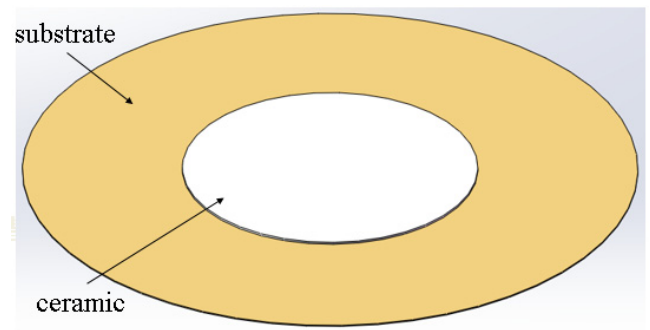

Fig. 2. Piezoelectric patch model

Table 1. Piezoelectric patches structure parameters $(\mathrm{mm})$

\begin{tabular}{|c|c|c|c|}
\hline Substrate diameter & Ceramic diameter & Substrate thickness & Ceramic thickness \\
\hline 15 & 11 & 0.15 & 0.2 \\
\hline 20 & 15 & 0.15 & 0.2 \\
\hline 25 & 18 & 0.15 & 0.2 \\
\hline 27 & 20 & 0.15 & 0.2 \\
\hline 35 & 20 & 0.15 & 0.2 \\
\hline 50 & 24 & 0.1 & 0.18 \\
\hline
\end{tabular}

From the Fig. 3, it can be seen that, the piezoelectric patch has obvious deformation after applying $200 \mathrm{~V}$ voltage on both ends of it, displacement of edge position is $0 \mu \mathrm{m}$, and in a central location to achieve maximum displacement about $190 \mu \mathrm{m}$.

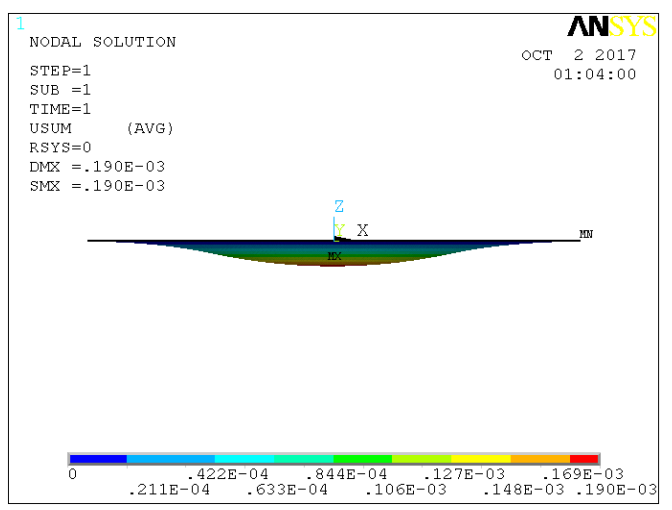

Fig. 3. Results of piezoelectric plate deformation

Fig. 4(a) shows the contour of piezoelectric patch deformation obtained by the surface fitting. The cavity volume change of different piezoelectric patches under $20 \mathrm{~V}$ voltage were compared, the result was shown in Fig. 4(b). The volume change increases with the increasing of the size of the piezoelectric patches and reached the maximum volume as $9.44 \mathrm{~mm}^{3}$ when diameter is $50 \mathrm{~mm}$. So, the larger piezoelectric patch can provide a larger volume change of the cavity. 


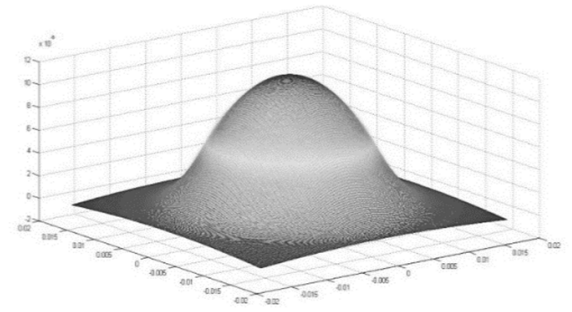

a) Result of surface fitting

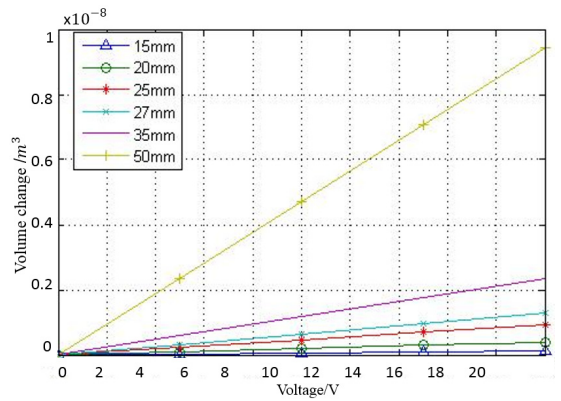

b) Comparison of simulation results

Fig. 4. Calculation of the volume variation of piezoelectric patches

\subsection{Square tube with piezoelectric acoustic liner}

The three-dimensional finite element method (FEM) was used to simulate the performance of piezoelectric acoustic liner. The simulation model is a rectangular pipe $(51 \mathrm{~mm} \times 51 \mathrm{~mm} \times 300 \mathrm{~mm})$ with piezoelectric acoustic liner (cavity: $\Phi=50 \mathrm{~mm}, h=1 \mathrm{~mm}$; neck: $\Phi=1 \mathrm{~mm}, h=2 \mathrm{~mm}$ ) on the wall of the pipeline (Fig. 5). The frequency-domain analysis is conducted to obtain the transmission loss.

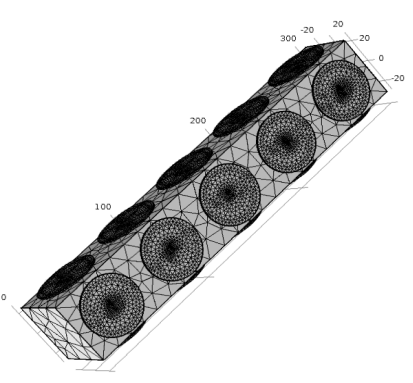

Fig. 5. FEM model

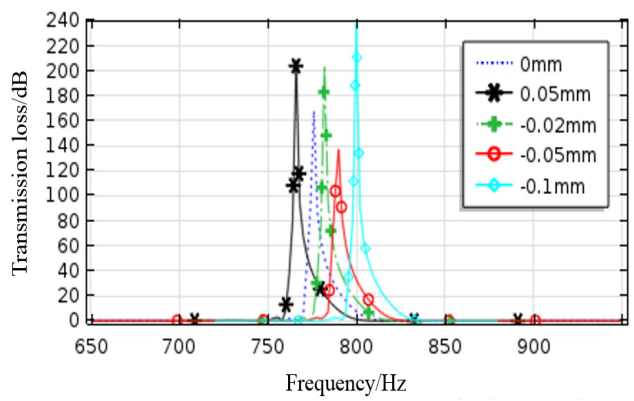

Fig. 6. Transmission loss curve

In the simulation process, piezoelectric patches deformation can be simulated by changing the height of the resonant cavity. It can be seen from the Fig. 6 that by changing the height of the chamber, the resonant frequency can be significantly offset.

\section{Experiment}

\subsection{Piezoelectric patch center displacement test}

The measurement of acoustic liner cavity volume variation is more complicated, this paper adopts the method of testing center displacement of piezoelectric patch, and the volume change of the cavity is calculated by fitting. The center displacement of piezoelectric patch was measured by laser micrometer, the test device is shown in the Fig. 7.

The center displacement of the piezoelectric patch with a diameter of $50 \mathrm{~mm}$ is measured in different shape parameters. Two types piezoelectric patches I and II were selected.

Table 2. Parameter of I and II (mm)

\begin{tabular}{|c|c|c|c|}
\hline Type & Substrate thickness & Ceramic thickness & Ceramic diameter \\
\hline I & 0.10 & 0.18 & 24 \\
\hline II & 0.10 & 0.12 & 30 \\
\hline
\end{tabular}


As shown in Fig.8 piezoelectric displacement test results for two kinds of parameters, $50 \mathrm{~mm}$ II type piezoelectric patch after the applied voltage of the variable is far greater than $50 \mathrm{~mm}$ I type. In $200 \mathrm{~V}$ DC voltage, II type piezoelectric patch center displacement can reach $450 \mu \mathrm{m}$, and only $200 \mu \mathrm{m}$ I type piezoelectric patch. The results show that by increasing the diameter of piezoelectric ceramic and reducing the thickness of piezoelectric ceramic, the shape deformation of piezoelectric patch can be increased.

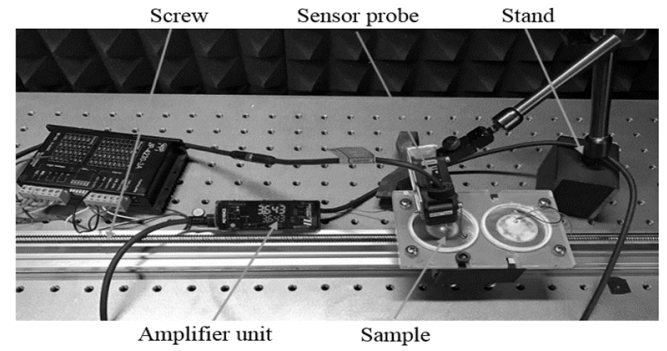

Fig. 7. Displacement test device diagram

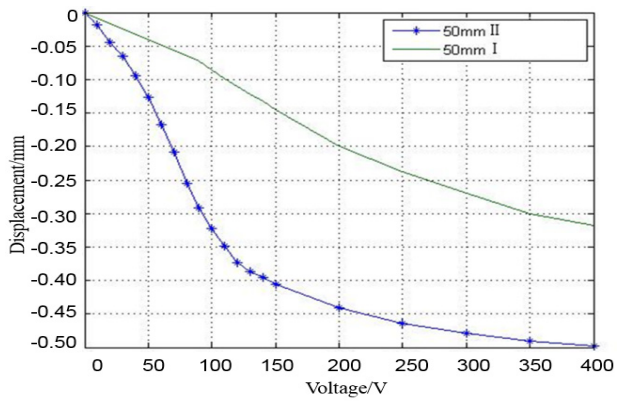

Fig. 8. Piezoelectric patch test result

\subsection{Acoustic performance test}

The experimental facility is shown in Fig. 9, it mainly includes the loudspeaker, the pipeline, the acoustic liner and the data acquisition device. By changing the DC voltage amplitude applied on the piezoelectric patches, the volume of the acoustic liner can be changed to alter its noise elimination frequency band.

In Fig. 10, the transmission loss measurement results show that when the voltage is applied, with the deformation of the piezoelectric patches, the resonant frequency has a distinct offset. It can be seen that the piezoelectric acoustic liner has a wide noise elimination frequency band, and its range is from $1100 \mathrm{~Hz}$ to $1300 \mathrm{~Hz}$ while the voltage range of $0 \mathrm{~V}$ to $200 \mathrm{~V}$.

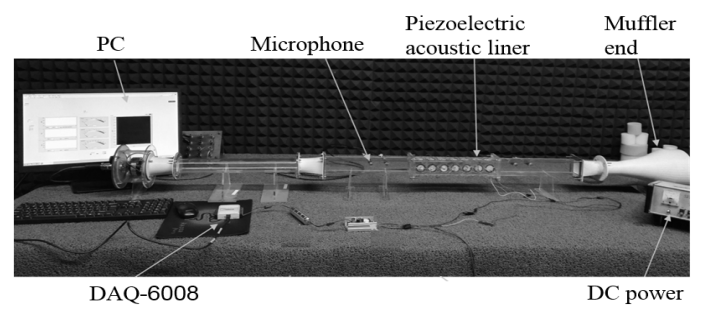

Fig. 9. Acoustic performance test device diagram

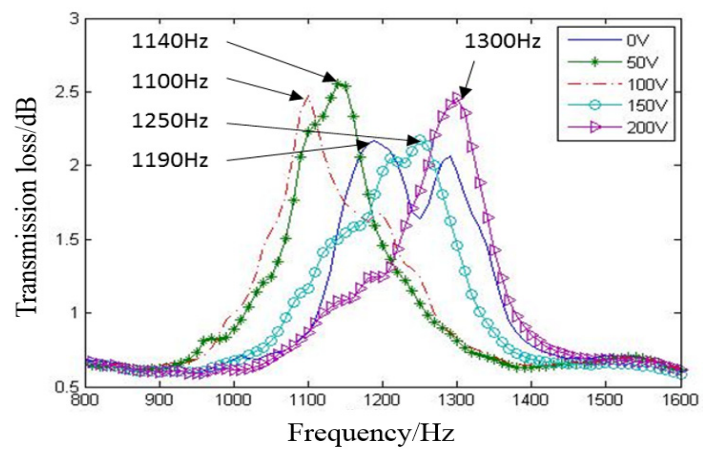

Fig. 10. Transmission loss measurement results 


\section{Conclusions}

The deformation of piezoelectric patches and the acoustic performance of piezoelectric acoustic liner are simulated and verified through experiment in this paper. The results show that when applying the $200 \mathrm{~V}$ DC voltage, the piezoelectric patches can realize the maximum center displacement changes of $450 \mu \mathrm{m}$, and the resonant frequency of the piezoelectric acoustic liner is offset by $110 \mathrm{~Hz}$, the broadband attenuation is realized and the noise elimination frequency band is broaden.

\section{Acknowledgement}

This work is supported by the National Nature Science Foundation of China (NSFC, Grant No. 51305409) and Natural Science Foundation of Shanxi (Grant No. 2013021020-2).

\section{References}

[1] Lv Haifeng, Pan Hongxia, Huang Jinying A design of tunable frequency resonant muffler. Journal of Vibration, Measurement and Diagnosis, Vol. 32, Issue 6, 2012, p. 1021-1025.

[2] Han Yannan, Lv Haifeng, Geng Yanzhang, et al. Design and optimization of the piezoelectric acoustic liner structure. Piezoelectrics and Acoustooptics, Vol. 39, Issue 2, 2017, p. 220-223.

[3] Wen Jianqiang, Zhang Liwang New advances in piezoelectric materials. Applied Acoustic, Vol. 32, Issue 5, 2013, p. 413-418.

[4] Wu Shengju, Zhang Mingduo The Principle and Method of Acoustic Measurement. Science Press, Beijing, 2014. 